\title{
Estrategias etnográficas para un encuadre innovador del COVID-19
}

\author{
Laura S. Teves ${ }^{1,3}$; Carolina Remorini 2,3, María Gabriela Morgante ${ }^{1,3}$ \\ ${ }^{1}$ Laboratorio de Investigaciones en Etnografía Aplicada - Facultad de Ciencias Naturales y \\ Museo, UNLP-CIC; ${ }^{2}$ Laboratorio de Investigaciones en Etnografía Aplicada - Facultad de Ciencias \\ Naturales y Museo, UNLP-CIC y CONICET; ${ }^{3}$ linea@fcnym.unlp.edu.ar
}

Resumen. Se aborda el problema del COVID-19, partiendo del concepto de sindemia y desde la perspectiva teórica y metodológica de una Etnografía Aplicada orientada a identificar factores que originan y/o modelan cambios en la vida cotidiana a micro escala, enfocando en facilitadores o barreras que dificultan el acceso a los recursos para el bienestar, la salud y la subsistencia. Nos interesa visibilizar procesos novedosos de cooperación y articulación social que reconocen a los actores e instituciones comprometidos en la atención y resolución de problemas en contextos locales. En este desarrollo se reconocen las demandas y necesidades específicas de los grupos vulnerables -personas con enfermedades preexistentes, personas mayores de 60 años, trabajadores esenciales de la salud, familias con personas COVID-19 positivos, entre otros-. Al respecto nos preocupa evaluar las dificultades que presentan diferentes sectores para resolver problemas cotidianos, como el acceso a servicios de salud y cuidado, teniendo en cuenta las redes de apoyo y las estrategias comunitarias locales.

Los objetivos que guiaron el proceso para abordar el problema del COVI-19 se fundamentan en la articulación de la investigación, extensión y transferencia. Estos objetivos aportan a la revisión de conceptos y enfoques desde la obtención de datos propios, resultando en el desarrollo de estrategias innovadoras. Nuestro desarrollo busca contribuir al fortalecimiento de las capacidades municipales y provinciales para el abordaje de la sindemia de COVID-19, a través de la construcción de herramientas, modelos y bases de datos sobre los efectos en la salud y el bienestar de diferentes sectores de la población local.

Nuestro desarrollo consiste en la generación de un Observatorio COVID-19, basado en el registro y sistematización de datos mixtos, el diseño de instrumentos, la construcción de bases de datos, y la modelización. El proyecto busca crear un Observatorio sobre COVID-19 con el objetivo de 
Innovación y Desarrollo Tecnológico y Social (2020) 2 (2): 306-335- Número especial COVID-19

relevar y transferir información novedosa sobre las redes personales, las perspectivas y experiencias de pacientes y trabajadores de salud, la circulación de recursos que contribuyan al cuidado y apoyo a grupos de vulnerables y situación de aislamiento por COVID-19 en el Gran La Plata. Los productos a desarrollar son un conjunto de herramientas para cumplir con los objetivos de relevar y transferir información novedosa sobre las redes personales, las perspectivas y experiencias de pacientes y trabajadores de salud, la circulación de recursos que contribuyan al cuidado y apoyo a grupos de vulnerables y la situación de aislamiento por COVID-19 en el Gran La Plata. Los productos son seis y se presentan a continuación:

Producto 1: Herramienta para una recolección estandarizada de datos de actores y relaciones en el contexto de prácticas cotidianas: de los *casos positivos de COVID-19, y de *recursos esenciales para el sostén cotidiano o red de apoyo y capital social; y *grupos vulnerables. Producto 2: Herramienta de relevamiento de datos sobre perspectivas y experiencias de los trabajadores de salud, investigadores y tomadores de decisiones. Producto 3: Herramienta de relevamiento de datos sobre perspectivas y experiencias cotidianas de las Personas Mayores en primera persona y las representaciones de otros integrantes de las unidades domésticas, especialmente de niños y niñas. Producto 4: Modelo de análisis reticular de COVID-19 basado en una selección de casos que incluya el cálculo del tamaño promedio de una red personal de los casos confirmados y de sus contactos estrechos, en muestras al azar. Así como la elaboración de materiales visuales gráficos que representen la dinámica de los contagios, en contexto. Producto 5: Base de datos y elaboración de cartografía temática de casos COVID-19 positivos y sus vínculos de contactos directos, incluyendo ubicación georeferenciada. Producto 6: Base de datos con información cualicuantitativa sobre políticas, guías e instrumentos de gestión local y de los otros países participantes. Análisis comparativo de políticas públicas.

Los productos obtenidos de esta propuesta podrán ser transferidos a profesionales de servicios e instituciones públicas, a gestores y tomadores de decisiones en políticas en salud, y a los referentes territoriales e institucionales en el AMBA. Consideramos que las innovaciones sociales que proponemos satisfacen simultáneamente las necesidades concretas de los grupos vulnerables, creando nuevas relaciones o colaboraciones sociales. Esto introduce cambios a nivel micro y macro estructurales, involucrando a las personas y sus comunidades de referencia e influyendo a nivel de las instituciones públicas y privadas, en sentido amplio. 
Palabras clave: etnografía aplicada; innovación social; transferencia; COVID-19; vida cotidiana; interacciones sociales; salud; bienestar

Recibido: 05/11/2020 Aceptado: 10/11/2020

DOI: https://doi.org/10.24215/26838559e026

\section{Ethnographic strategies for an innovative framework of COVID-19}

Abstract. The problem of Covid-19 is tackled from the concept of syndemic and the theoretical and methodological perspective of Applied Ethnography oriented to identify factors which cause or shape changes in everyday life at a micro scale, focusing on the facilitators or barriers that affect the access to the resources for welfare, health and subsistence. Likewise, we are interested in drawing attention to new cooperation and social articulation processes which acknowledge the actors and institutions committed to addressing and solving problems in local contexts.

In this development, the specific demands and needs of vulnerable groups are considered-people with pre-existing diseases, people over 60 years of age, healthcare workers, families with Covid-19 positive members, among others-. In this respect, we would like to assess the difficulties the different sectors present to solve everyday problems, such as the access to health care and services, taking the support nets and local community strategies into consideration.

The objectives that led the process to tackle the problem of Covid-19 are based on the articulation of the research, extension and transference. These objectives contribute to the revision of concepts and approaches by means of obtaining our own data, resulting in the development of innovative strategies. Our development seeks to contribute to the strengthening of municipal and provincial capacities to tackle Covid-19 syndemic, building tools, models and databases on the effects on health and welfare in the different sectors of the local population.

Our development is based on the generation of a Covid-19 Observatory according to the register and systematization of mixed data, instruments design, database construction and modelization. The project seeks to create an Observatory of Covid-19 with the purpose of collecting and transferring new information about personal nets, patients and healthcare workers' perspectives 
and experiences, the circulation of resources which contribute to the care and support of vulnerable groups and situations of isolation due to Covid-19 in the suburbs of La Plata.

The products to be developed are a set of tools to meet the objectives of collecting and transferring new information about personal networks, the perspectives and experiences of patients and health workers, the circulation of resources that contribute to the care and support of vulnerable groups and the situation of isolation due to COVID-19 in Greater La Plata. The products are six and are presented below: Product 1: tool for the standardized collection of data on actors and relationships in the context of everyday practices: on *Covid-19 positive cases, and on *essential resources for everyday support or support net and social capital; and *vulnerable groups. Product 2: tool for collection of data on healthcare workers, researchers and decision makers' perspectives and experiences. Product 3: tool for collection of data on elders' first hand representations, perspectives and everyday experiences and through other members of the family unit (especially boys and girls). Product 4: Covid-19 reticular analysis model based on the selection of cases which includes the standard size estimation of a personal net of confirmed cases and their close contacts, in random samples. Also, the elaboration of graphic material which represents infection dynamics in context. Product 5: database and elaboration of thematic cartography of Covid-19 positive cases and their bonds with direct contacts, including georeferenced location. Product 6: database with quantitative and qualitative information about politics, guides and management instruments, both local and from other participating countries. Comparative analysis of public policies.

The products obtained from this proposal can be transferred to professionals from public services and institutions, health policies managers and decision makers, and to territorial and institutional referents in the Metropolitan Area of Buenos Aires. We believe that the social innovations we propose simultaneously address the concrete needs of vulnerable groups, creating new social relationships and collaborations. This introduces changes at both micro and macro structural levels, which will involve reference people and their communities, and will have a comprehensive influence on both private and public institutions.

Keywords: applied ethnography; social innovation; transference; COVID-19; everyday life; social interactions; health; welfare 
Innovación y Desarrollo Tecnológico y Social (2020) 2 (2): 306-335- Número especial COVID-19

\section{Novedad u originalidad local en el conocimiento}

La pandemia de COVID-19 ha dado lugar a una situación sin precedentes a escala mundial, con más de un millón de muertos en la actualidad. La comprensión de sus dimensiones epidemiológicas, sanitarias, sociales, ambientales, políticas y económicas (Singer, 2003; Irons, 2020; Vanderslott y Marks, 2020; Vindrola y Johnson, 2020) requiere abordar su expresión a nivel local. La introducción del término sindemia permite su tratamiento como un fenómeno social que no se resuelve únicamente por la vía médica, y evidencia cómo un mismo evento tiene efectos dispares cuando se trata de contextos socio-ambientales caracterizados por la inequidad que, entre otros factores, aumenta la vulnerabilidad social (Singer y Claire, 2003; Singer et al 2017). Territorialidad, género, edad, ocupación, grado de acceso a la salud y educación son algunas de las variables fundamentales que merecen analizarse en la interseccionalidad con las expresiones de la pandemia y las posibilidades de atender a las recomendaciones en términos sanitarios.

La definición del problema, el enfoque analítico y la metodología desde una perspectiva etnográfica, permiten visibilizar cambios en la vida cotidiana a micro escala: barreras o facilitadores en el acceso a recursos para el bienestar, la salud y la subsistencia; así como formas novedosas de cooperación y articulación social. Igualmente favorece el reconocimiento de actores e instituciones locales, más o menos directamente comprometidos en la atención y resolución de problemas en contextos como el aquí descripto. 


\section{Grado de relevancia}

La relevancia del desarrollo propuesto reside en la aplicación de estrategias etnográficas al estudio de la cotidianeidad del COVID-19 a escala local. Ello condiciona la formulación de los objetivos conceptuales, metodológicos y de transferencia. Se vincula con un trabajo previo en los territorios a los que se aplica este desarrollo. La elección en término de política científica apuesta a trascender los espacios de laboratorio para construir agendas colaborativas. Se reconoce la necesidad de flexibilizar el diseño de herramientas, para adecuar el trabajo etnográfico, aplicado a las situaciones imprevistas que ofrece la dinámica de la pandemia (Valero y Morgante, 2020). También fortalece la perspectiva de la Universidad Nacional de La Plata de optimizar y potenciar sus recursos y generar resultados que atiendan a las especificidades locales. Por último, el desarrollo propuesto posibilita la creación de bases de datos en formatos seguros y accesibles, de acceso público.

Se reconocen como disciplinariamente relevantes las demandas y necesidades específicas de los conjuntos vulnerables -personas con enfermedades preexistentes, personas mayores de 60 años, trabajadores esenciales de la salud, entre otros-. Las dificultades que presentan diferentes sectores para resolver problemas de la vida cotidiana, entre ellos el acceso a servicios de salud y cuidado, exigieron reorientar las redes de apoyo hacia aquellas de tipo informal y otras estrategias comunitarias que las complementen. 
Desde una Etnografía Aplicada, es posible contribuir al abordaje de la sindemia articulando investigación, extensión y transferencia, aportando a la revisión de conceptos y enfoques desde datos propios resultado de la aplicación de estrategias innovadoras, algunas de las cuales se derivan de herramientas metodológicas clásicas de nuestro campo.

\section{Grado de pertinencia}

El objetivo es generar datos y modelos basados en variables específicas del contexto local, en este caso, el aglomerado urbano del Gran La Plata (Provincia de Buenos Aires), atendiendo a una población de más de 800.000 habitantes.

En el territorio bonaerense el manejo de la pandemia adquiere características distintivas respecto de otras regiones del país. Entre otras razones porque aquí reside cerca de un tercio de su población y se localiza el cordón peri-urbano más poblado, el Área Metropolitana de Buenos Aires (AMBA). Además, concentra el mayor porcentaje de Personas Mayores (de 60 años de edad y más), que se definen sanitariamente como uno de los grupos de riesgo y de mayor mortalidad.

Nuestro desarrollo busca contribuir al fortalecimiento de las capacidades municipales y provinciales para el abordaje del COVID-19, a través de la construcción de herramientas, modelos y bases de datos sobre los efectos en la salud y el bienestar de diferentes sectores de la población local. Ello podrá ser transferido a 
profesionales de servicios e instituciones públicas y a gestores y tomadores de decisiones en políticas públicas, referentes territoriales e institucionales.

Consideramos que esta innovación social satisface simultáneamente las necesidades territoriales y crea nuevas relaciones o colaboraciones sociales, que pueden implicar cambios estructurales en diferentes niveles, fundadas en las relaciones sociales y en las experiencias de los receptores (Moulaert et al, 2013; Comisión Europea, 2015; Bock, 2016; Jaeger-Erben et al, 2015; McMichael y Shipworth, 2013).

\section{Grado de demanda}

Siguiendo los principios de una Etnografía colaborativa, los primeros demandantes se identifican con los actores comunitarios con los que desarrollamos proyectos sostenidos en el tiempo, localizados en los barrios El Retiro, Villa Argüello y Etcheverry, y en la localidad de Melchor Romero de La Plata. La demanda se canalizó a través de distintas vías, entre las que se incluyen actores universitarios en la gestión territorial y la extensión, representantes en las mesas técnicas barriales y comités de crisis, agentes sanitarios, equipos docentes y orientadores pedagógicos, y miembros de otras organizaciones sociales, estatales o civiles.

La demanda derivó en la implementación de una primera línea de acciones sobre la base de la adecuación de intervenciones y proyectos preexistentes, que se 
evaluaron adecuados pero no suficientes para dar cuenta del impacto de la pandemia.

Una segunda instancia resultó en el desarrollo que aquí se expone, articulando líneas de trabajo y experticia de distintos investigadores del LINEA, tanto en el territorio bonaerense como en otras provincias, en diálogo con otros especialistas. Ello también dio lugar a abrir nuevas colaboraciones con actores universitarios y del sistema de salud local (Teves et al, 2018; Morgante et al, 2018; Remorini et al, 2020). Finalmente, en el mes de julio de 2020 la Universidad Nacional de La Plata, a través de MINERVA Incubadora de Emprendimientos, distinguió y promovió a este proyecto por su potencial para desarrollar ideas estratégicas en relación al COVID19, lo cual facilitó el testeo de los primeros pasos de las estrategias propuestas, su reformulación y la llegada a nuevos demandantes ${ }^{1}$.

\section{Desarrollo del producto}

Nuestro desarrollo consiste en la generación de un Observatorio COVID-19, basado en la construcción de datos de tipo mixto (cuali-cuantitativos) provenientes de un proyecto que articula investigación, extensión y transferencia.

\footnotetext{
1 "Redes personales, perspectivas y experiencias de pacientes y trabajadores de salud del Gran La Plata en el contexto de la pandemia de COVID-19. Estudio de asesoría rápida como aporte a las políticas públicas en el territorio bonaerense." LINEA-UNLP-CIC reconocido por la INCUBADORA MINERVA DE EMPRENDIMIENTOS como parte de los Proyectos estratégicos de la Universidad Nacional de La Plata, en el marco de la convocatoria "Tu idea Suma. COVID-19". Expediente Código 100 N 895/3/20 -Año 2014, La Plata, 7 julio de 2020.
} 
Innovación y Desarrollo Tecnológico y Social (2020) 2 (2): 306-335- Número especial COVID-19

Para su formulación partimos de los siguientes antecedentes:

- Si bien los datos sobre el COVID-19 hasta el momento son escasos y fragmentarios, existen numerosos antecedentes que demuestran la especificidad del abordaje antropológico y la metodología etnográficos para el análisis de las epidemias a lo largo de la historia reciente (Hewlett et al, 2005; Vanderslott, 2020; Vindrola et al, 2020).

- En el contexto actual, la necesidad de un enfoque que trascienda la visión estrecha de las pandemias como un problema médico, y de la salud como un proceso biológico, requiere de investigaciones que sean capaces de generar datos que aporten a una visión ecológica de la pandemia y den lugar a estrategias innovadoras para articular datos provenientes de los contextos locales.

- Las perspectivas históricas señalan la necesidad de considerar la pandemia como consecuencia de la coevolución de factores biológicos, políticos, comerciales y médicos (McNeal, 1970).

- El empleo del término "sindemia", acuñado por el antropólogo y médico Merrill Singer en 1990, resulta de relevancia para abordar la crisis originada por el brote de COVID-19. Desde esta perspectiva, se sostiene que los efectos sobre la salud ocasionados por el COVID-19 no pueden atribuirse únicamente a la acción del virus, y las infecciones no pueden considerarse de manera independiente del contexto socio- ambiental de cada persona. 
Innovación y Desarrollo Tecnológico y Social (2020) 2 (2): 306-335- Número especial COVID-19

- El COVID-19 y la medidas de aislamiento y distanciamiento social concomitantes pueden considerarse en términos de "acontecimiento disruptivo" en el curso de la cotidianeidad, provocando reelaboraciones en las estrategias de organización colectivas, ya sea a nivel doméstico, comunitario e institucional. Afrontar las situaciones desconocidas es posible sobre la base de la disponibilidad de un stock cultural, disponible y accesible al actor y que le sirve de referencia e interpretación para volver ante lo desconocido (Reguillo, 2005; Lalive D'Epinay, 2008).

- La escala y metodología etnográficas, con su foco en la la vida cotidiana y en los contextos relacionales, permiten articular datos sobre actividades, organización y vínculos en diferentes dominios de la vida colectiva, junto con mostrar la vulnerabilidad social a diferentes escalas y teniendo en cuenta sectores específicos que resultan de la interseccionalidad de variables socio-ambientales y epidemiológicas (Rugani, Caro, 2020; Bashir, Ben Jiang, Bilal et al, 2020; Morgante y Valero, 2020). Los datos sobre procesos a microescala pueden generar insights que den cuenta de las conexiones a escalas meso y macro en la estructura social.

- Desde una Etnografía Aplicada es posible contribuir al abordaje de la sindemia aportando a la revisión de conceptos y enfoques desde datos propios resultado de la aplicación de estrategias innovadoras. 
Con base en este enfoque y antecedentes, definimos nuestro proyecto y el desarrollo del producto, en función de reconocer un conjunto de problemas a diferentes niveles:

- La pandemia de COVID-19, desde el punto de vista de los estados y organismos internacionales de salud, ha generado la necesidad de contar con diagnósticos precisos y con datos confiables y actualizados sobre dimensiones epidemiológicas, sanitarias, socioambientales y económicas.

- El COVID-19 se contagia por contactos interpersonales, y no hay estudios sistemáticos en la Provincia de Buenos Aires sobre qué clase de flujos de contactos cotidianos depende esta dinámica, tomando la intersección de variables relevantes para la delimitación de contextos relacionales.

- El brote de COVID-19 y las medidas de aislamiento y distanciamiento social tienen efectos sobre el tamaño y estructura de las redes de apoyo, afectando su eficacia para vehiculizar diferentes tipos de recursos que aportan a la salud y bienestar material, físico o emocional de las personas. Esto obligó a reorientar el foco hacia las redes de apoyo de tipo informal y estrategias comunitarias que con frecuencia complementan e incluso sustituyen las redes de protección social y acceso a servicios universales de carácter formal (educación, salud y bienestar social) (Romero y Carbonell, 2020 citado en Morgante y Valero).

- Desde el inicio de la pandemia, los organismos públicos en Argentina formularon una serie de recomendaciones que fueron adquiriendo mayor focalización, no 
obstante prevalece una visión homogénea de los conjuntos sociales a los que se dirige, desconociendo la interseccionalidad de variables a escala local. Esto puso en escena la relevancia de contar con políticas públicas sensibles a las demandas y necesidades específicas de los conjuntos vulnerables (personas con enfermedades previas, las personas mayores de 60 años, trabajadores esenciales, particularmente quienes se desempeñan en instituciones de salud, en la detección, atención y/o cuidado de personas con COVID-19 u otras con necesidades especiales).

- Las recomendaciones y políticas oficiales propuestas por los organismos públicos en el marco de la vida cotidiana de las Personas Mayores se elaboran desde el perfil que representa sólo a una parcialidad. No obstante, se ha identificado un alto porcentaje de hogares con conformación unipersonal o unigeneracional vinculado con Personas Mayores, asociado a marcadores como: la dependencia a la asistencia para resolver las necesidad básicas, presencia de situaciones de violencia, o con roles y responsabilidades de cuidadores de niños, y con dificultades en el acceso a la tecnología (Encuesta Nacional sobre Calidad de Vida de Adultos Mayores 2012, Morgante, 2020).

- En el ámbito de las instituciones de salud, la declaración de la emergencia sanitaria² implicó adecuar los servicios y prestaciones a las necesidades de esta nueva realidad, con el propósito de garantizar la cobertura y atención a la población,

\footnotetext{
${ }^{2}$ Establecida por el Decreto de Necesidad y Urgencia 260/20206, que se llamó de aislamiento estricto, extendiéndose inicialmente del 20 de marzo al 12 de abril, en ese marco se toman medidas sanitarias para prevenir el avance del virus, que luego se fueron actualizando hasta el presente.
} 
focalizando en los grupos de riesgo. Este proceso puso de manifiesto diversos problemas preexistentes a la pandemia, a escala local, en cuanto a la organización y los recursos humanos y materiales del sector público.

- Las medidas sanitarias dieron lugar a recomendaciones y protocolos que se perciben como comunes y normativos de la actividad. No obstante, los trabajadores y tomadores de decisiones a nivel micro deben adaptar los mismos a sus contextos específicos, en función de recursos, saberes y valores locales, lo que permite anticipar acciones, barreras, facilitadores y resultados heterogéneos. La reorganización de espacios, tareas, roles y articulaciones entre trabajadores y servicios como parte de las estrategias de preparación y afrontamiento de la pandemia suponen una ruptura con respecto a rutinas de trabajo naturalizadas (Moglia et al, 2020; Sy et al, no publicado).

- Existen coincidencias y evidencias en los medios internacionales de lo que sucede a nivel global acerca de que la prestación de servicios y acciones de prevención, cuidado y atención de la salud ha generado una sobrecarga para el personal sanitario en diferentes niveles, incluyendo los psicosociales (San Juan et al, 2020; Vindrola et al, 2020).

En función de la necesidad de atender los aspectos mencionados de manera simultánea y complementaria, nos propusimos como objetivo general aportar a la revisión de conceptos y enfoques previos, que instruyan la obtención de datos 
Innovación y Desarrollo Tecnológico y Social (2020) 2 (2): 306-335- Número especial COVID-19

propios. Consecuentemente, la información resultante será utilizada para la construcción de modelos y bases de datos que instruyan y fundamenten el diseño de herramientas que sean aplicadas y validadas en situaciones que involucren a personas y grupos vulnerables desde el punto de vista de la salud, en el contexto sanitario del Covid-19.

Nuestro proyecto se basa en un diseño teórico y metodológico de tipo mixto, es decir que combina enfoques teórico-metodológicos y técnicas tales cómo las cualitativas clásicas de la etnografía, el análisis de redes sociales (ARS), el Paradigma Interdisciplinario del Curso de la Vida, la estadística, los sistemas de información geográfica (SIG) y los procedimientos de evaluación rápida (RAP).

Consideramos que las técnicas y métodos han sido eficaces en instancias de proyectos previos, pero que en este caso deben ser adaptados al problema y a los contextos actuales donde se aplicarán. Los instrumentos diseñados hasta el momento cuentan con la evaluación y supervisión del Comité de Ética de la UNLP que ha revisado los protocolos para el relevamiento de datos en pacientes y trabajadores de la salud. ${ }^{3}$

El planteo de nuestro proyecto parte de considerar la necesidad de aportar evidencia empírica sobre las dimensiones de los problemas mencionados anteriormente y en las características que adquieren en el ámbito local. En tanto no

\footnotetext{
${ }^{3}$ Aval y firma en conformidad del Comité Central Único de Bioética de la Universidad Nacional de La Plata con fecha y firma del 28 de agosto de 2020.
} 
existen estudios sistemáticos a nivel de la Provincia de Buenos Aires en general, y en particular de la Municipalidad de La Plata, que las aborden de manera integral, proponemos desarrollarlos a través de cuatro módulos interconectados, centrados en:

Módulo 1: Con el propósito de evaluar el alcance de las prácticas y los vínculos sociales incluidos en la categoría "circulación comunitaria" y/o la "transmisión por conglomerados", se espera identificar circuitos informales de contacto y prácticas sociales que habilitan canales de contagio comunitario usualmente sub-registrados. Para esto se propone la aplicación de una herramienta innovadora para la descripción, visualización y modelización de la red de contactos personales en pacientes diagnosticados como Covid-19 positivos, basado en el enfoque de ARS (Teves, 2020), focalizando en grupos de riesgo y zonas de vulnerabilidad del Gran La Plata. Los datos se obtendrán del Sistema Nacional de Vigilancia de la Salud (SNVS) y otras fuentes secundarias. Asimismo, a través del diseño y aplicación de una encuesta autoadministrada dirigida a pacientes y contactos, se podrá relevar información que complementará las bases de datos de salud preexistentes.

Módulo 2- Las medidas sanitarias de ASPO y DISPO suponen la pérdida o el aumento de relaciones personales, por lo tanto, la descripción de continuidades y cambios en la red de vínculos de apoyo, a través de la limitación a los contactos 
Innovación y Desarrollo Tecnológico y Social (2020) 2 (2): 306-335- Número especial COVID-19

habituales, requiere de monitoreo y ajustes para evitar la pérdida de relaciones. En este sentido, los recursos materiales y humanos pueden ser evaluados ante los cambios y eventos críticos en la vida cotidiana. En base a este diagnóstico, se propone la construcción de herramientas para abordar estos problemas mediante la descripción y análisis cuali-cuantitativa de las redes de apoyo y sus contextos, en base a la identificación del tipo y promedio de vínculos interpersonales. Asimismo, se propone relevar información sobre aspectos de la autopercepción de los actores sobre el aumento y/o disminución de su capital social, de salud y económico, en términos personales y comunitarios, en situación de COVID-19. La transferencia de estos resultados focaliza sobre sectores y familias vulnerables del Gran La Plata, visibilizando alternativas para acceder a recursos materiales, asistencia y cuidados, ante los aislamientos domiciliarios por casos de COVID-19 positivos.

Módulo 3- Se propone la creación de una base de datos que reúna información cualitativa sobre las percepciones y experiencias de efectores de salud pública de diferentes niveles de atención, así como de investigadores de la red de laboratorios de la UNLP para la detección de casos como parte de la Red Nacional de Covid-19. La información sobre estos aspectos será relevada mediante un instrumento adaptado a las instituciones adoptantes. A ello se suma la creación de una base de datos con información acerca de instrumentos y normativas que orientan la toma de decisiones y/o la planificación de la atención durante la pandemia de COVID-19, 
en base a información oficial disponible en Ministerios de Salud (nacionales y provinciales), organismos internacionales (OMS, OPS, otros) y de ciencia y tecnología. Se utilizarán estos datos para caracterizar micro contextos de trabajo y formas de organización, estructuras de colaboraciones o barreras entre los trabajadores de la salud para hacer frente a la pandemia, entre otros aspectos. A partir de ello se busca detectar el impacto en los procesos regulares de atención médica (incluida la atención para pacientes con COVID-19 y servicios que no atienden pacientes Covid-19), variaciones según servicio, efector y nivel de atención, variaciones de las políticas a lo largo del tiempo, implementación de políticas para abordar a largo plazo los efectos de Covid-19 en los servicios de salud.

Módulo 4- Partimos del supuesto de que las situaciones críticas, como en este caso la pandemia de COVID-19, pueden constituirse en oportunidades para la reformulación de representaciones sociales y sentidos que posibiliten reordenar la realidad cotidiana, provocando cambios en las concepciones previas y dando lugar a procesos de comunicación colectiva que colaboran en su tránsito. Para esto se propone realizar un trabajo colaborativo con las personas reconocidas como referentes territoriales, abordando de manera integral las problemáticas de Personas Mayores, y niños y niñas. Estas estrategias pueden resultar en la elaboración de diagnósticos participativos que sean tomados como insumos para identificar las variables que definen los problemas y contextos de vulnerabilidad 
Innovación y Desarrollo Tecnológico y Social (2020) 2 (2): 306-335- Número especial COVID-19

locales. En este sentido, focalizamos sobre la relevancia de los relatos en términos de la posibilidad de acceso a la salud, incidencia y atención a las recomendaciones sanitarias, atención a la presencia de nuevos vínculos y redefinición de anteriores, y a la dinámica de las relaciones intergeneracionales. Asimismo consideramos necesario transferir estos resultados por medio de una "traducción" de la información dirigida a la comunidad, a través de formas de comunicación significativa facilitada mediante dispositivos tecnológicos y multimediales.

El desarrollo de cada módulo según objetivos y actividades específicas generará un volumen de datos mixtos provenientes de instrumentos diversos, que deberán sistematizarse, almacenarse y disponerse de manera tal que puedan ser apropiados por diversos sectores. De este modo, la meta final de nuestro proyecto es el desarrollo de un producto (Observatorio COVID-19) que representa una novedad local en términos de su organización y tipo de datos. La generación de un Observatorio Covid-19 como el propuesto, requiere de la construcción y actualización permanente de datos cuali y cuantitativos, en formatos accesibles y flexibles que puedan ser utilizados por diferentes actores de la comunidad y organismos gubernamentales. Asimismo requiere del monitoreo permanente del manejo de la información asumiendo los recaudos legales y éticos de la protección de datos sensibles, tal como lo establece la normativa a nivel nacional e internacional. 
La construcción de datos a partir de la información relevada en los diferentes módulos del proyecto, para ser incluida dentro del Observatorio, requiere del desarrollo de productos intermedios que consisten en instrumentos, protocolos, mapas y bases de datos cuali-cuantitativos, algunos de los cuales detallamos a continuación:

Producto 1: Herramienta para una recolección estandarizada de datos de actores y relaciones en el contexto de prácticas cotidianas: de los *casos positivos de COVID19, y de *recursos esenciales para el sostén cotidiano o red de apoyo y capital social; $y$ *grupos vulnerables.

Producto 2: Herramienta de relevamiento de datos sobre perspectivas y experiencias de los trabajadores de salud, investigadores y tomadores de decisiones.

Producto 3: Herramienta de relevamiento de datos sobre perspectivas y experiencias cotidianas de las Personas Mayores en primera persona; y de las representaciones de otros integrantes de las unidades domésticas, especialmente de niños y niñas.

Producto 4: Modelo de análisis reticular de COVID-19 basado en una selección de casos que incluya el cálculo del tamaño promedio de una red personal de los casos confirmados y de sus contactos estrechos, en muestras al azar. Así como la elaboración de materiales visuales gráficos que representen la dinámica de los contagios, en contexto. 
Producto 5: Base de datos y elaboración de cartografía temática de casos COVID-19 positivos y sus vínculos de contactos directos, incluyendo ubicación georeferenciada.

Producto 6: Base de datos con información cuali-cuantitativa sobre políticas, guías e instrumentos de gestión local y de los otros países participantes. Análisis comparativo de políticas públicas.

Los productos obtenidos de esta propuesta podrán ser transferidos a profesionales de servicios e instituciones públicas, a gestores y tomadores de decisiones en políticas en salud, y a los referentes territoriales e institucionales en el AMBA. Consideramos que las innovaciones sociales que proponemos satisfacen simultáneamente las necesidades concretas de los grupos vulnerables, creando nuevas relaciones o colaboraciones sociales.

\section{Financiamiento}

El proyecto actualmente no dispone de recursos específicos para las próximas instancias de desarrollo. Estamos a la espera de adoptantes para su financiación y ampliación de otras instancias de transferencia.

\section{Agradecimientos}

Deseamos expresar nuestro agradecimiento al conjunto de personas e instituciones que promovieron y/o participan de este proyecto. A la Facultad de Ciencias 
Innovación y Desarrollo Tecnológico y Social (2020) 2 (2): 306-335- Número especial COVID-19

Naturales y Museo de la UNLP y a la Comisión de Investigaciones Científicas de la Provincia de Buenos Aires, de las que depende el Laboratorio de Investigaciones de Etnografía Aplicada, al que pertenecemos las autoras. En particular, a todos los integrantes del equipo LINEA-UNLP-CIC que colaboraron para desarrollar la idea proyecto y trabajar intensamente en el relevamiento de datos y análisis, reconociendo el potencial de nuestro equipo para adaptarnos a las demandas de la situación. A las comunidades de los barrios El Retiro, Villa Argüello y Etcheverry, y de la localidad de Melchor Romero de La Plata, con quienes desarrollamos proyectos previos. Al Dr. Gonzalo Javier Márquez por las gestiones académicocientíficas entre la UNLP y las instituciones de salud adoptantes del proyecto y por estimularnos a presentar la idea la Incubadora MINERVA-UNLP. A Minerva por avalar la "Idea Proyecto" destacando la importancia de las innovaciones sociales para abordar el estudio de la pandemia. AI Comité de Bioética de la UNLP por su evaluación del proyecto y recomendaciones. A los directivos y personal de las instituciones de salud y red de laboratorios de la UNLP por promover el proyecto, facilitar su ejecución, por disponer de su tiempo y compartir sus experiencias. Al Dr. Javier Díaz y su equipo del CESPI por el asesoramiento para sistematizar, organizar y visibilizar la información. A la Dra Cecilia Vindrola-Padros del RREAL (Rapid Reseach Evaluation and Appraisal Lab) de University College London (UCL) por invitarnos a formar parte del estudio "A rapid appraisal of healthcare workers' perceptions of care delivery in the context of the COVID-19 pandemic", por su asesoramiento y por 
Innovación y Desarrollo Tecnológico y Social (2020) 2 (2): 306-335- Número especial COVID-19

facilitarnos protocolos que utilizamos como base para los instrumentos diseñados adaptados al contexto local. Por último, a la Dra Elisa Bellotti Centro Mitchel, Univ. Manchester por mantener el compromiso de colaboración científico-académica con la UNLP.

\section{Referencias bibliográficas}

Bashir, M. F., Ma, B. J., Bilal, Komal, B., Bashir, M. A., Farooq, T. H., Iqbal, N., y Bashir, M. (2020). Correlation between environmental pollution indicators and COVID-19 pandemic: A brief study in Californian context. Environmental research, 187, 109652. https://doi.org/10.1016/j.envres.2020.109652

Beebe J (1995). Basic concepts and techniques of rapid appraisal. Human Organization 54(1): 42-51.

Christakis, N. A., y Fowler, J. H. (2007). The spread of obesity in a large social network over 32 years. The New England journal of medicine, 357(4), 370-379. https://doi.org/10.1056/NEJMsa066082

Ennis-McMillan, M. y Hedges, K. (2020). Pandemic Perspectives: Responding to COVID-19. Open Anthropology, 8

https://www.americananthro.org/Staylnformed/OAArticleDetail.aspx?ItemNumber $=25631$

Gale, N. K., Heath, G., Cameron, E., Rashid, S., y Redwood, S. (2013). Using the framework method for the analysis of qualitative data in multi-disciplinary health 
research. BMC medical research methodology, 13, 117. https://doi.org/10.1186/1471-

\section{8-13-117}

Green, J. y Thorogood, N. (2013). Qualitative methods for health research. London: SAGE.

Harris, K.J., Jerome, N.W., Fawcett, S.B. (1997). Rapid assessment procedures: A review and critique. Human Organization 56(3): 375-378. https://doi.org/10.17730/humo.56.3.w525025611458003

Hernández-Ascanio, J., Tirado-Valencia, P., Ariza-Montes, A. (2016). El concepto de innovación social: ámbitos, definiciones y alcances teóricos. CIRIEC-España, Revista de Economía Pública, Social y Cooperativa, 88, (diciembre, 2016), 164-199 Centre International de Recherches et d'Information sur l'Economie Publique, Sociale et Coopérative Valencia, España. https://www.redalyc.org/pdf/174/17449696006.pdf Hewlett, B. S., Epelboin, A., Hewlett, B. L., Formenty, P. (2005). Medical anthropology and Ebola in Congo: Cultural models and humanistic care. Bulletin de la Société de pathologie exotique, 98(3), 230-236.

Imai, H., Matsuishi, K., Ito, A., Mouri, K., Kitamura, N., Akimoto, K., Mino, K., Kawazoe, A., Isobe, M., Takamiya, S., y Mita, T. (2010). Factors associated with motivation and hesitation to work among health professionals during a public crisis: a cross sectional study of hospital workers in Japan during the pandemic (H1N1) 2009. BMC public health, 10, 672. https://doi.org/10.1186/1471-2458-10-672 
Innovación y Desarrollo Tecnológico y Social (2020) 2 (2): 306-335- Número especial COVID-19

Irons R. (2020). Pandemic ... or syndemic? Re-framing COVID-19 disease burden and 'underlying health conditions'. Social anthropology : the journal of the European Association of Social Anthropologists, $10.1111 / 1469-8676.12886$.

\section{https://doi.org/10.1111/1469-8676.12886}

Ives, J., Greenfield, S., Parry, J. M., Draper, H., Gratus, C., Petts, J. I., Sorell, T., y Wilson, S. (2009). Healthcare workers' attitudes to working during pandemic influenza: a qualitative study. BMC public health, 9, 56. https://doi.org/10.1186/1471-2458-9-56 Jaeger-Erben M., Offenberger, U. (2014). A Practice Theory Approach to Sustainable Consumption. GAIA Ecological Perspectives on Science and Society 23(3):166-174. https://doi.org/10.14512/gaia.23.S1.4

Koh, Y., Hegney, D. G., y Drury, V. (2011). Comprehensive systematic review of healthcare workers' perceptions of risk and use of coping strategies towards emerging respiratory infectious diseases. International journal of evidence-based healthcare, 9(4), 403-419. https://doi.org/10.1111/j.1744-1609.2011.00242.x

Lalive D'epinay, C. (2008) La vida cotidiana: Construcción de un concepto sociológico $\begin{array}{lllll}\text { y antropológico. } & \text { Sociedad } & \text { Hoy, } & 14, & \text { 9-31. }\end{array}$ https://www.redalyc.org/pdf/902/90215158002.pdf

Latour, B. (1983) Dadme un laboratorio y levantaré el Mundo. Publicación original: "Give Me a Laboratory and I will Raise the World", en: K. Knorr-Cetina y M. Mulkay (eds.), Science Observed: Perspectives on the Social Study of Science, Londres: Sage, 1983, pp. 141-170. 
Innovación y Desarrollo Tecnológico y Social (2020) 2 (2): 306-335- Número especial COVID-19

Linstrom, M. y Marais, W. (2012). Qualitative news frame analysis: A methodology. Communitas 17: 21-38. https://journals.ufs.ac.za/index.php/com/article/view/991

Maunder, R. G., Lancee, W. J., Mae, R., Vincent, L., Peladeau, N., Beduz, M. A., Hunter, J. J., y Leszcz, M. (2010). Computer-assisted resilience training to prepare healthcare workers for pandemic influenza: a randomized trial of the optimal dose of training. BMC health services research, 10, 72. https://doi.org/10.1186/1472-6963-10$\underline{72}$

McNeal, W.H. (2003). Plagues and Peoples. Siglo XXI.

Michie, S. (2020). Behavioural science must be at the heart of the public health response to covid-19, https://blogs.bmj.com/bmj/2020/02/28/behavioural-sciencemust-be-at-the-heart-of-the-public-health-response-to-covid-19/

Moglia, B., Derossi, P.D., Aragunde, G.E., Sy, A. (2020). Transformaciones en los espacios de atención a la salud durante la emergencia sanitaria. Un análisis exploratorio de las narrativas de los trabajadores. XIV Jornadas Nacionales de Debate Interdisciplinario de Salud y Población "Salud, derechos y desigualdades: desafíos urgentes". 2, 3 y 4 sept. Buenos Aires: Argentina Mora, J. (2020). Los discursos de la pandemia paralela. Semanario Universidad, México. https://semanariouniversidad.com/cultura/los-discursos-de-la-pandemiaparalela/ 
Innovación y Desarrollo Tecnológico y Social (2020) 2 (2): 306-335- Número especial COVID-19

Morgante, M.G. y Valero, A. (2020) Coronavirus y vejeces en Argentina. Geronte. Revista de Estudios sobre Procesos de la Vejez; 7.

\section{http://sedici.unlp.edu.ar/handle/10915/96879}

Morgante, M.G. (2020). Etnografía aplicada a las vejeces en el contexto de la pandemia (Argentina 2020). En: Aportes de la Mesa de Trabajo de Personas Mayores de la Universidad Nacional de La Plata en el contexto de la pandemia de COVID-19. http://sedici.unlp.edu.ar/handle/10915/96568

Morgante, M.G.; Remorini, C.; Teves, L. 2018. Prácticas de investigación y extensión en el Departamento de Molinos (Salta, Argentina): Reflexiones en torno al trabajo sostenido en territorio. Conclusiones de las VI Jornadas de Extensión de MERCOSUR. I Coloquio Regional de la Reforma Universitaria: 2018 / Daniel Eduardo Herrero. 1a ed. Tandil: Universidad Nacional del Centro de la Provincia de Buenos Aires, 2018. http://extension.unicen.edu.ar/jem/subir/uploads/2018 548.docx

Moulaert, F., MacCallum, D. y Hillier, J (2013). Social innovation: Intuition, precept, concept, theory and practice. En: Moulaert, F., MacCallum, D., Mehmood, A., y Hamdouch, A. (Eds.).The International Handbook on Social Innovation Collective Action, Social Learning and Transdisciplinary Research. Cheltenham, UK: Edward Elgar Publishing. https://doi.org/10.4337/9781849809993.00011

OCDE (2015)- Frascati Manual 2015: Guidelines for Collecting and Reporting Data on Research and Experimental Development, The Measurement of Scientific, 
Innovación y Desarrollo Tecnológico y Social (2020) 2 (2): 306-335- Número especial COVID-19

Technological and Innovation Activities. Publicado por acuerdo con la OCDE, París (Francia). http://dx.doi.org/10.1787/9789264239012-en

Raven, J., Wurie, H. y Witter, S. (2018). Health workers' experiences of coping with the Ebola epidemic in Sierra Leone's health system: a qualitative study. BMC Health Serv Res 18, 251. https://doi.org/10.1186/s12913-018-3072-3

Reguillo, R. (2005). La construcción simbólica de la ciudad: sociedad, desastre y comunicación. México: Ed. Iteso.

Remorini, C., Teves, L.; Pasarin, L. y Palermo, M.L. (2020). Etnografía y salud rural: trayectorias de investigación en los Valles Calchaquíes, Argentina. Anthropologica. Dossier "Antropología Médica y de la Salud: aportes desde el Sur Global". http://revistas.pucp.edu.pe/index.php/anthropologica

Rugani, B. y Caro, D. (2020). Impact of COVID-19 outbreak measures of lockdown on the Italian Carbon footprint, Science of The Total Environment, 737, 139806 https://doi.org/10.1016/j.scitotenv.2020.139806

Sams, K., Desclaux, A., Anoko, J., Akindès, F., Egrot, M., Sow, K., Taverne, B., Bila, B., Cros, M., Keïta-Diop, M., Fribault, M.,y Wilkinson, A. (2017). From Ebola to Plague and Beyond: How Can Anthropologists Best Engage Past Experience to Prepare for New Epidemics? Society of Cultural Anthropology Member Voices, Fieldsights, December 7. https://culanth.org/fieldsights/from-ebola-to-plague-and-beyondhow-can-anthropologists-best-engage-past-experience-to-prepare-for-newepidemics 
Innovación y Desarrollo Tecnológico y Social (2020) 2 (2): 306-335- Número especial COVID-19

San Juan, V., Aceituno, D., Djellouli, N., Sumray, K., Regenold, N., Syversen, A., Symmons, S., Dowrick, A., Mitchinson, L., Singleton, G. y Vindrola-Padros, C. (2020). Healthcare Workers' Mental Health and Wellbeing During the COVID-19 Pandemic in the UK: Contrasting Guidelines with Experiences in Practice. medRxiv 2020.07.21.20156711. https://doi.org/10.1101/2020.07.21.20156711

Smith, J., y Firth, J. (2011). Qualitative data analysis: the framework approach. Nurse researcher, 18(2), 52-62. https://doi.org/10.7748/nr2011.01.18.2.52.c8284

Singer, M., y Clair, S. (2003). Syndemics and public health: reconceptualizing disease in bio-social context. Medical anthropology quarterly, 17(4), 423-441. https://doi.org/10.1525/maq.2003.17.4.423

Singer, M., Bulled, N., Ostrach, B., y Mendenhall, E. (2017). Syndemics and the biosocial conception of health. Lancet (London, England), 389(10072), 941-950. https://doi.org/10.1016/S0140-6736(17)30003-X

Sy, A.; Moglia, B. y Derrosi, P. (no publicado). Todo se transformó completamente" atención a la pandemia de COVID-19 desde la perspectiva de los trabajadores de la salud.

Teves, L.; Pasarin L.; Cueto J.J; D’Abramo, S.; Castro, F. (2018). Mapeo de redes sociales en contextos de vulnerabilidad y riesgo ambiental en el Área Gran La Plata. Observatorio Medioambiental La Plata. Informe final. Observatorio medioambiental La Plata UNLP. http://omlp.sedici.unlp.edu.ar/dataset/subproyecto-a-ii-mapeo-deredes-sociales-en-contextos-de-vulnerabilidad-y-riesgo-ambiental 
Innovación y Desarrollo Tecnológico y Social (2020) 2 (2): 306-335- Número especial COVID-19

Valero, A. y Morgante, M.G. (2020). Reformulando estrategias de intervención universitaria con Personas Mayores: Proyectos de vida y pandemia. Geronte. Revista de estudios sobre procesos de la vejez, 10.

Vanderslott, S. (2020). Neglected Tropical Diseases. Nature and Culture, 15 (1): 78110. https://doi.org/10.3167/nc.2020.150105

Vanderslott S. y Marks T. (2020). Health diplomacy across borders: The case of yellow fever and COVID-19. Journal of Travel Medicine, 27 (5), taaa112. https://doi.org/10.1093/jtm/taaa112

Vanderslott, S. y Marks, T. (2020) Travel restrictions as a disease control measure: Lessons from yellow fever. Global Public Health. https://doi.org/10.1080/17441692.2020.1805786

Vindrola-Padros, C., Chisnall, G., Cooper, S., Dowrick, A., Djellouli, N., Symmons, S. M., Martin, S., Singleton, G., Vanderslott, S., Vera, N., y Johnson, G. A. (2020). Carrying Out Rapid Qualitative Research During a Pandemic: Emerging Lessons From COVID19. Qualitative health research, 30(14), 2192-2204. https://doi.org/10.1177/1049732320951526 